\title{
Pseudospin-electron model at weak coupling
}

\author{
I.V.Stasyuk, T.S.Mysakovych \\ Institute for Condensed Matter Physics \\ of the National Academy of Sciences of Ukraine, \\ 1 Svientsitskii Str., 79011 Lviv, Ukraine
}

Received May 17, 2002

The static and dynamic dielectric susceptibility of the pseudospin-electron model is investigated in the random phase approximation. The possibility of the phase transition to the homogeneous phase, to the phase with doubly modulated lattice period and to the incommensurate phase is revealed in the regime $\mu=$ const depending on the value of the chemical potential. The phase separation on the homogeneous and phase with doubly modulated lattice period is established and the phase diagrams $(n, h)$ are built. The influence of the tunnelling-like splitting on the phase transition picture is studied. The presence of pseudospin-wave excitations and the smooth excitation spectrum and the possibility of their superimposing are revealed.

Key words: pseudospin-electron model, phase transitions, excitation spectrum

PACS: $63.20 . R y, 64.70 . K b, 71.10 . F d, 77.80 . B h$

\section{Introduction}

The pseudospin-electron model (PEM) appeared in connection with the investigation of the role of the the locally anharmonic structure elements in the high temperature superconductors (HTSC) in the formation of their electron spectrum and pairing correlations which lead to the appearance of a superconducting state (the crystals of $\mathrm{YBaCuO}$-type structure). The electron subsystem in PEM is described by the Hubbard Hamiltonian whereas the interaction of the electrons with local anharmonic mode of lattice vibrations is treated using pseudospin formalism. The model Hamiltonian looks as follows:

$$
\begin{aligned}
H= & \sum_{i}\left[U n_{i, \uparrow} n_{i, \downarrow}-\mu\left(n_{i, \uparrow}+n_{i, \downarrow}\right)+g S_{i}^{z}\left(n_{i, \uparrow}+n_{i, \downarrow}\right)-h S_{i}^{z}-\Omega S_{i}^{x}\right] \\
& +\sum_{i, j, \sigma} t_{i j} c_{i, \sigma}^{\dagger} c_{j, \sigma}
\end{aligned}
$$


where the electron transfer $(t$-term), electron correlation $(U$-term) and pseudospinelectron interaction ( $g$-term) are included; energy of the local (tunnelling-like) level splitting $(\Omega$-term) and asymmetry of the local anharmonic potential $(h$-term) are also taken into account [1-4].

The investigations of this model in the recent years were devoted to the analysis of the structure of the electron spectrum with an allowance for strong short range correlations [5], to the investigation of the charge and pseudospin correlations and dielectric susceptibility $[6,7]$ as well as to the pair correlations of the superconducting type [3]. The PEM thermodynamics was the subject of consideration as well. The attention was paid to the equilibrium and metastable states and bistability phenomena [8], the phase transitions between states with the different pseudospin orientation and electron concentration in the regime of a fixed electron chemical potential [9], the transitions into the phase separated states at a given value of the average electron concentration [10]. The simplified PEM (at $\Omega=0$ and $t=0$ but with the inclusion of the direct pseudospin-pseudospin interaction) was the subject of consideration in [9]. The possibility of the first order phase transitions between uniform states and the tendency to the phase separation were established. Similar results were obtained for the case $t \neq 0, \Omega=0$ at the large values of the interaction constant $g$ both in the limit $U \rightarrow \infty$ for the two-sublattice PEM [11] and for the simplified version of the model at $U=0[12,13]$. The investigation [12] was carried out in the framework of the dynamic mean-field theory (DMFT) approach that is exact in the limit of the infinite dimensionality of space; in the paper [13] the thermodynamically consistent scheme of the generalized random phase approximation (GRPA) [14] was used. In the case $g \gg W$ ( $W$ is a half-width of the electron energy band) the electron band is always split and this defines the peculiarities of the system behaviour. The possibility of the appearance of the doubly modulated (the so-called chess-board) phase was shown [13] for the simplified PEM $(U=0, \Omega=0)$ in this case. Phase diagrams which describe the transitions between homogeneous and modulated phases at the change of the field $h$, the electron chemical potential $\mu$ and the temperature $T$ were built.

The PEM has a relation to the known in literature and intensively investigated in recent times Falicov-Kimball (FK) model [15], in which the interaction between the localized and the moving particles (electrons) is taken into account. The presence of homogeneous, chess-board and incommensurately modulated phases, the transitions between them as well as the possibility of the phase separation were revealed for this model [16-20] using the dynamic mean field approximation. The existence of the phase transition from the uniform to the chess-board phase in the regime of the fixed electron chemical potential $\mu_{e}$ and fixed concentration of localized particles $\rho_{i}$ was shown in the limit $d \rightarrow \infty$ [16]. The phase diagram built by J.Freericks [17]. manifests that an incommensurate phase can be stable at intermediate values of $\mu_{e}$ in the case of a weak interaction and disappears at the increase of the coupling constant. To track the first-order phase transition to the segregated phase, the behaviour of Helmholz free energy was studied in [20] in the cases of the hypercubic and Bethe lattices. In the regime of the fixed itinerant particle concentration $\rho_{e}$ the transition 
to the chess-board phase was continuous (a second order phase transition). The phase separation diagrams $\left(T, \rho_{e}\right),\left(T, \rho_{i}\right)$ were built including both strong and weak coupling cases.

The simplified PEM corresponds directly to the FK model in the case when the interaction between electrons and tunnelling-like splitting in the PEM are absent and when the localized and the moving particles in FK model have different chemical potentials. But even in this situation, the regimes of thermodynamic averaging are different in both cases (a fixed concentration of the localized particles for FK model and a given value of the field $h$ for the PEM). In general case, the PEM is not equivalent to FK model and thus thermodynamics of the PEM can possess its own features.

The aim of this paper is to investigate the thermodynamically stable states of the system, described by the PEM at $U=0$ in the case of weak coupling, when the interaction constant $g$ is small in comparison with the width of the electron band $2 W$ (it was shown in [12] that the electron band does not split in this case). The behaviour of dielectric susceptibility will be analysed for this purpose. The features of excitation spectrum of the PEM will be the subject of investigation as well.

The thermodynamics of the PEM in the case of weak coupling was considered for uniform and chess-board phase in the mean field approximation in our previous paper [21]. It was shown that the system can undergo the first order phase transition between uniform phases and the first or the second order phase transition from the uniform phase to the phase with doubly modulated lattice period. The $(h, \mu),(T, h)$ phase diagrams were built, and the phase separation for the uniform case was investigated. However, some problems of thermodynamics (such as the possibility of transition into the incommensurate state, the phase separation with the participation of the chess-board phase) and dynamics (the spectrum of collective excitations) were out of attention in [21]. These problems are considered in the present paper.

The dielectric susceptibility in the dipole approximation is determined by the Green's function constructed of the operators of the electric dipole momentum. In the case of isothermal response

$$
\chi_{\mathrm{T}}\left(\vec{q}, \omega_{n}\right)=\int_{0}^{\beta}\left\langle T_{\tau} M(0) M(\tau)\right\rangle_{\vec{q}} \mathrm{e}^{\mathrm{i} \omega_{n} \tau} \mathrm{d} \tau-\beta\langle M\rangle^{2} \delta\left(\omega_{n}\right)
$$

it is expressed in terms of the Matsubara Green's function. The so-called "isolated" response is described by means of the two-time Zubarev Green's function

$$
\chi_{\mathrm{I}}(\vec{q}, \omega) \sim\langle\langle M \mid M\rangle\rangle_{\vec{q} \omega} .
$$

The dipole momentum of the unit cell can be taken in the form [4]

$$
M_{i}=d_{e} n_{i}+d_{\mathrm{s}} S_{i}^{z},
$$

where the electron contribution arising due to nonhomeopolarity of filling of the electron orbitals is taken into account besides the pseudospin contribution (connected with the displacement of an anharmonic ion). In crystals with the structure of the 
high- $T_{\mathrm{c}}$ superconductor $\mathrm{YBa}_{2} \mathrm{Cu}_{3} \mathrm{O}_{7-\delta}$ type the expression (4) corresponds to the transverse component of polarization (this component is connected with the motion of the apex oxygen ions along the direction normal to the $\mathrm{Cu}-\mathrm{O}$ layers [4]).

\section{Random phase approximation}

At first we will calculate the isothermal susceptibility $\chi_{\mathrm{T}}$ using diagrammatic approach. Consideration of the model in the weak coupling approximation will be carried out similarly to the traditional investigation of the weak one-site correlation $U$ in the Hubbard model. In the case $U \ll W$ the Hartree-Fock approximation can be applied as a zero-order one, using the decoupling

$$
U n_{\sigma} n_{-\sigma} \rightarrow U\left[n_{\sigma}\left\langle n_{-\sigma}\right\rangle+n_{-\sigma}\left\langle n_{\sigma}\right\rangle-\left\langle n_{\sigma}\right\rangle\left\langle n_{-\sigma}\right\rangle\right]
$$

It makes it possible to satisfactorily describe the magnetic properties of the Hubbard model in the case when the correlational splitting of the electron band is absent (see, for example [22]). For the simplified PEM with $U=0$ the interaction constant $g$ plays the role which is similar to that of the energy $U$ in the Hubbard model. At the decrease of the constant $g$ below a critical value $(g \sim W)$ the system should pass to the mean field regime of the Hartree-Fock type. The analysis performed in the framework of the DMFT shows that in this case the gap between the two energy subbands in the electron spectrum disappears.

Based on the above mentioned arguments we use the approximation

$$
g n_{i} S_{i}^{z} \rightarrow g n_{i}\left\langle S_{i}^{z}\right\rangle+g\left\langle n_{i}\right\rangle S_{i}^{z}-g\left\langle n_{i}\right\rangle\left\langle S_{i}^{z}\right\rangle,
$$

constructing the zero-order Hamiltonian. Here the pseudospin-electron interaction is taken into account in the spirit of the mean field approximation (MFA) through an internal self-consistent field which acts on electrons and pseudospins.

The initial Hamiltonian can be rewritten as a sum

$$
\begin{aligned}
H & =H_{0}+H_{\mathrm{int}}, \\
H_{0} & =H_{\mathrm{el}}+H_{\mathrm{s}}+U, \\
H_{\mathrm{el}} & =\sum_{i, \sigma}(g \eta-\mu) n_{i, \sigma}+\sum_{i, j, \sigma} t_{i j} c_{i, \sigma}^{\dagger} c_{j, \sigma}, \\
H_{\mathrm{s}} & =\sum_{i}\left[(g n-h) S_{i}^{z}+\Omega S_{i}^{x}\right], \\
U & =-g \sum_{i} n \eta=-N g n \eta, \\
H_{\mathrm{int}} & =\sum_{i}\left(n_{i \uparrow}+n_{i \downarrow}-n\right)\left(S_{i}^{z}-\eta\right) .
\end{aligned}
$$

The unperturbed Hamiltonian $H_{0}$ can be diagonalized by means of the transition to $k$-representation and using the transformation

$$
S_{i}^{z}=\sigma_{i}^{z} \cos \theta+\sigma_{i}^{x} \sin \theta
$$




$$
\begin{aligned}
S_{i}^{x}= & \sigma_{i}^{x} \cos \theta-\sigma_{i}^{z} \sin \theta, \\
\sin \theta=\Omega / \lambda, & \lambda=\sqrt{(g\langle n\rangle-h)^{2}+\Omega^{2}} .
\end{aligned}
$$

As a result,

$$
\begin{aligned}
H_{0} & =-\lambda \sum_{i} \sigma_{i}^{z}-g N n \eta+\sum_{k, \sigma}\left(g \eta-\mu+t_{k}\right) n_{k \sigma}, \\
H_{\text {int }} & =g \sum_{i} \frac{1}{N} \sum_{\sigma k k^{\prime}} \mathrm{e}^{\mathrm{i}\left(k-k^{\prime}\right) R_{i}}\left(c_{k \sigma}^{+} c_{k^{\prime} \sigma}-n\right)\left(S_{i}^{z}-\eta\right) .
\end{aligned}
$$

To calculate the susceptibility $\chi_{\mathrm{T}}$ we use an approximation which is analogous to the random phase approximation (RPA), which is usually applied in the case of pseudospin systems with the direct interaction between pseudospins, or to the generalized random phase approximation (GRPA) elaborated at the consideration of the strongly correlated electron systems. As an example, first of all we will calculate the Green's function $\left\langle T_{\tau} S_{l}^{z}(\tau) S_{l^{\prime}}^{z}\left(\tau^{\prime}\right)\right\rangle$, where the operators are in the Heisenberg representation $A(\tau)=\mathrm{e}^{\tau H} A \mathrm{e}^{-\tau H}$. Using the standard representation of the $\exp (-\beta H)$ operator

$$
\mathrm{e}^{-\beta H}=\mathrm{e}^{-\beta H_{0}} \sigma(\beta), \quad \sigma(\beta)=T \exp \left(-\int_{0}^{\beta} H_{\text {int }}(\tau) \mathrm{d} \tau\right)
$$

we obtain the following expression for the function $\left\langle T_{\tau} S^{z}(\tau) S^{z}\left(\tau^{\prime}\right)\right\rangle$

$$
\begin{aligned}
& \left\langle T_{\tau} S^{z}(\tau) S^{z}\left(\tau^{\prime}\right)\right\rangle=\frac{1}{\langle\sigma(\beta)\rangle_{0}}\left\langle T_{\tau} S^{z}(\tau) S^{z}\left(\tau^{\prime}\right) \sigma(\beta)\right\rangle_{0} \\
& \left\langle T_{\tau} S^{z}(\tau) S^{z}\left(\tau^{\prime}\right) \sigma(\beta)\right\rangle_{0}^{\mathrm{c}}=\cos ^{2} \theta\left\langle T_{\tau} \sigma^{z}(\tau) \sigma^{z}\left(\tau^{\prime}\right) \sigma(\beta)\right\rangle_{0}^{\mathrm{c}}+\sin ^{2} \theta\left\langle T_{\tau} \sigma^{x}(\tau) \sigma^{x}\left(\tau^{\prime}\right) \sigma(\beta)\right\rangle_{0}^{\mathrm{c}} \\
& \quad+\sin \theta \cos \theta\left\langle T_{\tau} \sigma^{z}(\tau) \sigma^{x}\left(\tau^{\prime}\right) \sigma(\beta)\right\rangle_{0}^{\mathrm{c}}+\sin \theta \cos \theta\left\langle T_{\tau} \sigma^{x}(\tau) \sigma^{z}\left(\tau^{\prime}\right) \sigma(\beta)\right\rangle_{0}^{\mathrm{c}}
\end{aligned}
$$

Here the operators are given in the interaction representation $A(\tau)=\mathrm{e}^{\tau H_{0}} A \mathrm{e}^{-\tau H_{0}}$, the averaging $\langle\ldots\rangle_{0}$ is performed over the distribution with $H_{0}$, the symbol $\langle\ldots\rangle_{0}^{c}$ denotes the separation of the connected diagrams. We will do an expansion in powers of $H_{\text {int }}$ :

$$
\begin{aligned}
\left\langle T_{\tau} \sigma_{l}^{\alpha}(\tau) \sigma_{l^{\prime}}^{\beta}\left(\tau^{\prime}\right)\right\rangle & =\left\langle T_{\tau} \sigma_{l}^{\alpha}(\tau) \sigma_{l^{\prime}}^{\beta}\left(\tau^{\prime}\right)\right\rangle_{0}-\int_{0}^{\beta} \mathrm{d} \tau_{1}\left\langle T_{\tau} \sigma_{l}^{\alpha}(\tau) \sigma_{l^{\prime}}^{\beta}\left(\tau^{\prime}\right) H_{\text {int }}\left(\tau_{1}\right)\right\rangle_{0} \\
+ & \frac{1}{2 !} \int_{0}^{\beta} \mathrm{d} \tau_{1} \int_{0}^{\beta} \mathrm{d} \tau_{2}\left\langle T_{\tau} \sigma_{l}^{\alpha}(\tau) \sigma_{l^{\prime}}^{\beta}\left(\tau^{\prime}\right) H_{\text {int }}\left(\tau_{1}\right) H_{\text {int }}\left(\tau_{2}\right)\right\rangle_{0}-\ldots
\end{aligned}
$$

Calculating the mean values of the $T_{\tau}$-products of the pseudospin and the electron operators we use the diagrammatic techniques based on the Wick's theorem for the spin operatoes [23] (besides the usual procedure for the Fermi operators). After elimination in this way of the nondiagonal $\sigma^{ \pm}$operators we perform the semi-invariant expansion in order to calculate the mean values of the remaining products of the $\sigma^{z}$ operators. 
We use the following unperturbed Green's functions

$$
\begin{aligned}
\left\langle T_{\tau} \sigma_{l}^{+}(\tau) \sigma_{l^{\prime}}^{-}\left(\tau^{\prime}\right)\right\rangle_{0} & =-2\left\langle\sigma^{z}\right\rangle_{0} K_{l l^{\prime}}^{0}\left(\tau-\tau^{\prime}\right), \\
\left\langle T_{\tau} c_{k \sigma}(\tau) c_{k^{\prime} \sigma}^{+}\left(\tau^{\prime}\right)\right\rangle_{0} & =\delta_{k k^{\prime}} G_{k \sigma}^{0}\left(\tau-\tau^{\prime}\right)
\end{aligned}
$$

and semi-invariants [23]:

$$
\begin{aligned}
\left\langle T_{\tau} \sigma_{l}^{z}(\tau) \sigma_{l^{\prime}}^{z}\left(\tau^{\prime}\right)\right\rangle_{0} & =b^{2}+b^{\prime} \delta_{l l^{\prime}} \\
\left\langle T_{\tau} \sigma_{l}^{z}(\tau) \sigma_{l^{\prime}}^{z}\left(\tau^{\prime}\right) \sigma_{l^{\prime \prime}}^{z}\left(\tau^{\prime \prime}\right)\right\rangle_{0} & =b^{3}+b b^{\prime}\left(\delta_{l l^{\prime}}+\delta_{l^{\prime} l^{\prime \prime}}+\delta_{l l^{\prime \prime}}\right)+b^{[2]} \delta_{l l^{\prime}} \delta_{l l^{\prime \prime}}
\end{aligned}
$$

etc. Here

$$
b=b(\beta \lambda)=\left\langle\sigma_{l}^{z}\right\rangle_{0}=\frac{1}{2} \tanh \frac{\beta \lambda}{2} ; \quad b^{\prime}=\frac{\partial b}{\partial(\beta \lambda)} ; \quad b^{[n]}=\frac{\partial^{n} b}{\partial(\beta \lambda)^{n}} .
$$

In the diagrammatic notations

$$
\begin{aligned}
& K_{l l^{\prime}}^{0}\left(\tau-\tau^{\prime}\right): \quad \frac{\tau}{\mathrm{l}}-+-\frac{\tau^{\prime}}{\mathrm{l}^{\prime}} ; \quad G_{k \sigma}^{0}\left(\tau-\tau^{\prime}\right): \frac{\tau \underset{\mathrm{k} \sigma}{\mathrm{C}} \tau^{\prime}}{;} \\
& \left\langle\sigma_{l^{\prime}}^{z}\right\rangle_{0}: \quad \text { ○ ; } \quad\left\langle\sigma_{l}^{z}(\tau) \sigma_{l^{\prime}}^{z}\left(\tau^{\prime}\right)\right\rangle_{0}^{\mathrm{c}}: \overbrace{\mathrm{I} \mathrm{I}^{\prime}}^{\bigodot^{\tau^{\prime}}} .
\end{aligned}
$$

After the transition to the $\omega, k$-representation, where

$$
\frac{1}{\beta} \sum_{n} \mathrm{e}^{-\mathrm{i} \omega_{n}\left(\tau_{1}-\tau_{2}\right)} \frac{1}{N} \sum_{k} \mathrm{e}^{\mathrm{i} k\left(R_{l}-R_{i}\right)} K_{k}^{0}\left(\omega_{n}\right)=K_{l i}^{0}\left(\tau_{1}-\tau_{2}\right),
$$

and

$$
\begin{aligned}
G_{k \sigma}^{0} & =\frac{1}{i \omega_{n}-t_{k}-g \eta+\mu} \\
K_{q}^{0}\left(\omega_{n}\right) & =\frac{1}{\mathrm{i} \omega_{n}-\lambda} .
\end{aligned}
$$

At the summation of diagrams for the functions (10) we restrict ourselves in the spirit of the RPA (or GRPA) to diagrams having a structure of multi-loop chains. It should be noted that the usage of the MFA Hamiltonian as the zero-order one leads to the compensation of diagrams, which possess the "single-tail" inclusions (having a Caylee-tree structure and consisting of the electron loops) into the Green's function lines and into ovals, corresponding to semi-invariants. The corresponding mean-field corrections are taken into account in the zero-order functions $K^{0}, G^{0}$ and semi-invariants $b^{[n]}$.

At the consideration of the second order terms for the $\left\langle T_{\tau} \sigma_{l}^{+}(\tau) \sigma_{l^{\prime}}^{-}\left(\tau^{\prime}\right)\right\rangle$ function we consider only the diagrams of the following type: 


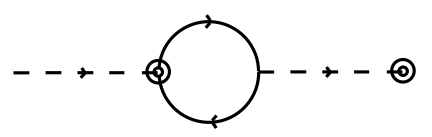

with the contribution

$$
\frac{1}{2} \sin ^{2} \theta g^{2} K_{q}^{0}\left(\omega_{n}\right)\left\langle\sigma_{z}\right\rangle_{0} \Pi_{q}\left(\omega_{n}\right) K_{q}^{0}\left(\omega_{n}\right)\left\langle\sigma_{0}\right\rangle_{0} .
$$

Here we have introduced the notation:

$$
\Pi_{q}\left(\omega_{n}\right)=\frac{1}{\beta N} \sum_{\omega_{n_{1}} k_{1}} G_{k_{1} \sigma}^{0}\left(\omega_{n 1}\right) G_{k_{1}-q, \sigma}^{0}\left(\omega_{n_{1}}-\omega_{n}\right) .
$$

The diagrams of the forth order, which we take into account, are as follows: such a diagram

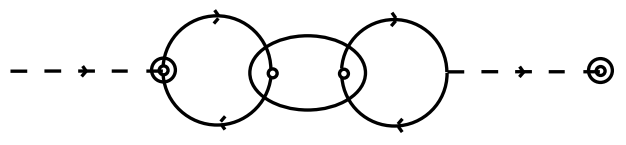

appears due to the term

$$
\left\langle T_{\tau} \sigma_{l}^{+}(\tau) \sigma_{l^{\prime}}^{-}\left(\tau^{\prime}\right) \sigma_{i_{1}}^{+}\left(\tau_{1}\right) \sigma_{i_{2}}^{-}\left(\tau_{2}\right) \sigma_{i_{3}}^{z}\left(\tau_{3}\right) \sigma_{i_{4}}^{z}\left(\tau_{4}\right)\right\rangle,
$$

and these two diagrams

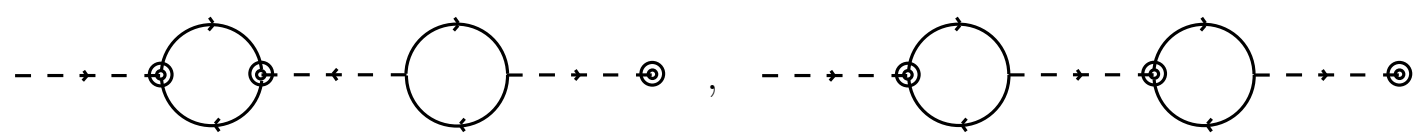

appear due to the term

$$
\left\langle T_{\tau} \sigma_{l}^{+}(\tau) \sigma_{l^{\prime}}^{-}\left(\tau^{\prime}\right) \sigma_{i_{1}}^{+}\left(\tau_{1}\right) \sigma_{i_{2}}^{-}\left(\tau_{2}\right) \sigma_{i_{3}}^{+}\left(\tau_{3}\right) \sigma_{i_{4}}^{-}\left(\tau_{4}\right)\right\rangle .
$$

The resulting contribution of the forth order is given by the following expression

$$
\frac{1}{2} \sin ^{2} \theta g^{4} K^{0}(+)\left\langle\sigma_{z}\right\rangle_{0} \Sigma \Pi K^{0}(+)\left\langle\sigma_{z}\right\rangle_{0}
$$

here

$$
K^{0}(+) \equiv K_{q}^{0}\left(\omega_{n}\right), \quad K^{0}(-) \equiv K_{q}^{0}\left(-\omega_{n}\right), \quad M \equiv M_{q}\left(\omega_{n}\right)=\beta b^{\prime} \cos ^{2} \theta \delta\left(\omega_{n}\right) .
$$

We have introduced here the irreducible part

$$
\Sigma=\sin ^{2} \theta \frac{K^{0}(+)+K^{0}(-)}{2}\left\langle\sigma^{z}\right\rangle_{0}-M
$$

The junctions between electron loops are realized by semi-invariants or by the boson Green's functions $K^{0}(+), K^{0}(-)$.

The contribution of the sixth order where the diagrams of the above considered type with three electron loops are taken into account is equal to

$$
\frac{1}{2} \sin ^{2} \theta g^{6} K^{0}(+)\left\langle\sigma^{z}\right\rangle_{0} \Pi \Sigma \Pi \Sigma \Pi K^{0}(+)\left\langle\sigma^{z}\right\rangle_{0} .
$$


As a result, we will have the following series:

$$
\begin{aligned}
K_{q}^{+-} & =-\frac{1}{2}\left\langle T \sigma^{+} \sigma^{-}\right\rangle_{q} \\
& =\frac{1}{2} \sin ^{2} \theta g^{2} K^{0}(+)\left\langle\sigma^{z}\right\rangle_{0} \Pi\left(1+g^{2} \Sigma \Pi+g^{4}(\Sigma \Pi)^{2}+\ldots\right) K^{0}(+)\left\langle\sigma^{z}\right\rangle_{0}
\end{aligned}
$$

Its sum is equal to

$$
K_{q}^{+-}=\frac{1}{2} \sin ^{2} \theta g^{2} K^{0}(+)\left\langle\sigma^{z}\right\rangle_{0} \Pi \frac{1}{1-g^{2} \sum \Pi} K^{0}(+)\left\langle\sigma^{z}\right\rangle_{0}
$$

The series (19) is similar to that obtained in the RPA in the case of a direct electronelectron interaction [6]; the semi-invariants and boson functions play a role of the vertices joining the electron loops. In the case of the PEM with the direct pseudospin interaction the latter have a meaning of the zero-order irreducible parts which are connected one with another by the interaction lines.

Similarly we obtain the expressions for functions $K_{q}^{-+}\left(\omega_{n}\right), K_{q}^{++}\left(\omega_{n}\right), K_{q}^{--}\left(\omega_{n}\right)$. In general

$$
K_{q}^{\alpha \beta}=\frac{1}{2} \sin ^{2} \theta g^{2} K^{0}(\alpha)\left\langle\sigma^{z}\right\rangle_{0} \Pi \frac{1}{1-g^{2} \Sigma \Pi} K^{0}(-\beta)\left\langle\sigma^{z}\right\rangle_{0} .
$$

Thus, we can find the Green's function $\left\langle T \sigma^{x} \sigma^{x}\right\rangle_{q}=-2 K_{q}^{x x}\left(\omega_{n}\right)$; here

$$
\begin{aligned}
K_{q}^{x x}\left(\omega_{n}\right)= & \frac{1}{4}\left(K^{++}+K^{--}+K^{-+}+K^{+-}\right) \\
= & \frac{1}{4}\left[K^{0}(+)+K^{0}(-)\right]\left\langle\sigma^{z}\right\rangle_{0} \\
& +\frac{1}{2} \sin ^{2} \theta g^{2} \frac{K^{0}(+)+K^{0}(-)}{2}\left\langle\sigma^{z}\right\rangle_{0} \frac{\Pi}{1-g^{2} \Sigma \Pi} \frac{K^{0}(+)+K^{0}(-)}{2}\left\langle\sigma^{z}\right\rangle_{0} .
\end{aligned}
$$

In a similar way we can find the functions $\left\langle T \sigma^{+} \sigma^{z}\right\rangle,\left\langle T \sigma^{z} \sigma^{z}\right\rangle,\left\langle T \sigma^{-} \sigma^{z}\right\rangle$ and thereafter obtain the expressions for $K^{x z}$ and $K^{z z}$ :

$$
\begin{aligned}
K^{x z} & =-\frac{1}{2} \tan \theta g^{2} \frac{K^{0}(+)+K^{0}(-)}{2}\left\langle\sigma^{z}\right\rangle_{0} \frac{\Pi}{1-g^{2} \Sigma \Pi} M, \\
K^{z z} & =-\frac{1}{2} \frac{M}{\cos ^{2} \theta}+\frac{1}{2 \cos ^{2} \theta} g^{2} M \frac{\Pi}{1-g^{2} \Sigma \Pi} M .
\end{aligned}
$$

Finally, we can write the correlator $\left\langle T S^{z} S^{z}\right\rangle_{q}$ :

$$
\left\langle T S^{z} S^{z}\right\rangle_{q \omega_{n}}=-\frac{\Sigma}{1-g^{2} \Sigma \Pi_{q}}
$$

This expression can be interpreted as a solution of the equation

$$
-\left\langle T S^{z} S^{z}\right\rangle_{q \omega_{n}} \equiv \mathfrak{G}=\Sigma+\Sigma g^{2} \Pi_{q} \mathfrak{G}
$$


which is analogous to the Larkin equation for the system with a direct interaction $-J_{i j} S_{i}^{z} S_{j}^{z}$. The factor $g^{2} \Pi_{q}$ plays the same role as an interaction $\beta J_{q}$ in the latter case. Explicit expressions for an effective interaction function $\Pi_{q}(\omega)$ and irreducible part $\Sigma(\omega)$ are the following (here the analytical continuation $i \omega_{n} \rightarrow \omega$ is performed)

$$
\begin{aligned}
\Pi_{q} & =\frac{1}{N} \sum_{k} \frac{n\left(t_{k}\right)-n\left(t_{k-q}\right)}{\omega+t_{k}-t_{k-q}} \\
\Sigma & =\sin ^{2} \theta \frac{\lambda\left\langle\sigma^{z}\right\rangle_{0}}{\omega^{2}-\lambda^{2}}-\beta b^{\prime} \cos ^{2} \theta \delta(\omega), \\
b^{\prime} & =\left(\frac{1}{4}-\left\langle\sigma^{z}\right\rangle_{0}^{2}\right) .
\end{aligned}
$$

In the case $\omega=0$, the correlator $\left\langle T_{\tau} S^{z} S^{z}\right\rangle$ is given by the expression

$$
\left\langle T S^{z} S^{z}\right\rangle_{q}=\frac{\lambda\left\langle\sigma^{z}\right\rangle_{0} \sin ^{2} \theta+\lambda^{2} \beta b^{\prime} \cos ^{2} \theta}{-\lambda^{2}-g^{2} \Pi_{q} \sin ^{2} \theta \lambda\left\langle\sigma^{z}\right\rangle_{0}-\lambda^{2} g^{2} \Pi_{q} \beta b^{\prime} \cos ^{2} \theta} .
$$

Let us calculate now the isolated susceptibility $\chi_{\mathrm{I}}(q, \omega)(3)$ in the same approximation. We can use, as usual, the equation of motion method for the two-time Green's functions. At first, writing the equation for the function $\left\langle\left\langle\sigma_{i}^{+} \mid \sigma_{l}^{-}\right\rangle\right\rangle$we will find the commutator

$$
\begin{aligned}
{\left[\sigma_{i}^{+}, H\right]=} & -g\left(n_{i \uparrow}+n_{i \downarrow}\right) \cos \theta \sigma_{i}^{+}+g\left(n_{i \uparrow}+n_{i \downarrow}\right) \sin \theta \sigma_{i}^{z}+\Omega \cos \theta \sigma_{i}^{z}+\Omega \sin \theta \sigma_{i}^{+} \\
& +h \cos \theta \sigma_{i}^{+}-h \sin \theta \sigma_{i}^{z} .
\end{aligned}
$$

After decoupling in the spirit of the Tyablikov approximation we have

$$
\left\langle\left\langle\left[\sigma_{i}^{+}, H\right] \mid \sigma_{i}^{-}\right\rangle\right\rangle=\left\langle\left\langle\lambda \sigma_{i}^{+}+g \sin \theta\left(n_{i \uparrow}+n_{i \downarrow}\right)\left\langle\sigma^{z}\right\rangle \mid \sigma_{l}^{-}\right\rangle\right\rangle .
$$

Here the relation $\left\langle\left\langle\sigma_{i}^{z} \mid \sigma_{l}^{-}\right\rangle\right\rangle=0$ is taken into account; it follows from the fact that in the adopted approximation $\left[\sigma_{i}^{z}, H\right]=0$.

The Green's function $\left\langle\left\langle n_{i \uparrow}+n_{i \downarrow} \mid \sigma_{l}^{-}\right\rangle\right\rangle$can be found using the following procedure. Let us write the equation for the function $\left\langle\left\langle c_{i \uparrow}^{+} c_{j \uparrow} \mid \sigma_{l}^{-}\right\rangle\right\rangle$. Having separated, at the decoupling of the higher order Green's function, the pseudospin and electron degrees of freedom we have

$$
\begin{aligned}
\left\langle\left\langle c_{i \uparrow}^{+} c_{j \uparrow} \mid \sigma_{l}^{-}\right\rangle\right\rangle= & g\left\langle c_{i \uparrow}^{+} c_{j \uparrow}\right\rangle \sin \theta\left\langle\left\langle\left(\sigma_{j}^{x}-\sigma_{i}^{x}\right) \mid \sigma_{l}^{-}\right\rangle\right\rangle \\
& +\sum_{k} t_{j k}\left\langle\left\langle c_{i \uparrow}^{+} c_{k \uparrow} \mid \sigma_{l}^{-}\right\rangle\right\rangle-\sum_{k} t_{i k}\left\langle\left\langle c_{k \uparrow}^{+} c_{j \uparrow} \mid \sigma_{l}^{-}\right\rangle\right\rangle .
\end{aligned}
$$

After the transition to $k$-representation one can obtain the following expression:

$$
\left\langle\left\langle c_{k_{1} \uparrow}^{+} c_{k_{2} \uparrow} \mid \sigma_{k_{3}}^{-}\right\rangle\right\rangle=\frac{1}{\sqrt{N}} \frac{\left(n_{k_{1} \uparrow}-n_{k_{2} \uparrow}\right) g \sin \theta}{\hbar \omega-t_{k_{2}}+t_{k_{1}}}\left\langle\left\langle\sigma^{x} \mid \sigma^{-}\right\rangle\right\rangle_{k_{3}} \delta\left(k_{2}-k_{1}+k_{3}\right) .
$$

Respectively, we can write for $\left\langle\left\langle n_{i \uparrow} \mid \sigma_{l}^{-}\right\rangle\right\rangle$:

$$
\left\langle\left\langle n_{i \uparrow} \mid \sigma_{l}^{-}\right\rangle\right\rangle=\frac{1}{N} \sum_{k_{3}} \mathrm{e}^{-\mathrm{i} k_{3}\left(R_{i}-R_{l}\right)} \Pi_{k_{3}}(\omega) g \sin \theta\left\langle\left\langle\sigma^{x} \mid \sigma^{-}\right\rangle\right\rangle_{k_{3}} .
$$


Returning to the Green's function $\left\langle\left\langle\sigma_{i}^{+} \mid \sigma_{l}^{-}\right\rangle\right\rangle$and using (29) we have the equation:

$$
\hbar \omega\left\langle\left\langle\sigma^{+} \mid \sigma^{-}\right\rangle\right\rangle_{q}=\frac{\hbar}{2 \pi}\left\langle\sigma^{z}\right\rangle+\lambda\left\langle\left\langle\sigma^{+} \mid \sigma^{-}\right\rangle\right\rangle_{q}+g^{2} \sin ^{2} \theta\left\langle\sigma^{z}\right\rangle \Pi_{q}(\omega)\left\langle\left\langle\sigma^{x} \mid \sigma^{-}\right\rangle\right\rangle_{q} .
$$

In a similar way one can find the equation for the Green's function $\left\langle\left\langle\sigma_{i}^{-} \mid \sigma_{l}^{-}\right\rangle\right\rangle$:

$$
\hbar \omega\left\langle\left\langle\sigma^{-} \mid \sigma^{-}\right\rangle\right\rangle_{q}=-\lambda\left\langle\left\langle\sigma^{-} \mid \sigma^{-}\right\rangle\right\rangle_{q}-g^{2} \sin ^{2} \theta\left\langle\sigma^{z}\right\rangle \Pi_{q}(\omega)\left\langle\left\langle\sigma^{x} \mid \sigma^{-}\right\rangle\right\rangle_{q}
$$

From the equations (33) and (34) we can find functions $\left\langle\left\langle\sigma^{x} \mid \sigma^{x}\right\rangle\right\rangle_{q}$ and $\left\langle\left\langle S^{z} \mid S^{z}\right\rangle\right\rangle_{q}=$ $\sin ^{2} \theta\left\langle\left\langle\sigma^{x} \mid \sigma^{x}\right\rangle\right\rangle_{q}$. Finally

$$
\left\langle\left\langle S^{z} \mid S^{z}\right\rangle\right\rangle_{q}=\frac{\sin ^{2} \theta\left\langle\sigma^{z}\right\rangle \lambda}{\omega^{2}-\lambda^{2}-\lambda g^{2} \sin ^{2} \theta\left\langle\sigma^{z}\right\rangle \Pi_{q}(\omega)} .
$$

The decoupling procedure used herein is equivalent to the random phase approximation in diagrammatic method for the systems with direct interaction. The obtained result is similar to the one (expression (24)) for the Matsubara Green's function but differs from it due to the absence of the terms proportional to $\delta(\omega)$. It is a manifestation of the difference between the isolated $\chi_{\mathrm{I}}$ and isothermal $\chi_{\mathrm{T}}$ susceptibilities of the PEM and is characteristic of Ising-type models with a transverse field [24].

In a similar way, (using the equation of motion method and a decoupling procedure) we can find the Green's functions $\langle\langle n \mid n\rangle\rangle_{q},\left\langle\left\langle n \mid S^{z}\right\rangle\right\rangle_{q}$ and $\left\langle\left\langle S^{z} \mid n\right\rangle\right\rangle_{q}$. Thus the susceptibility $\chi_{\mathrm{I}}$ can be written as follows:

$$
\chi_{\mathrm{I}}=d_{e}^{2}\left(\frac{h}{2 \pi} \Pi_{q}+4 g^{2} \Pi_{q}^{2}\left\langle\left\langle S^{z} \mid S^{z}\right\rangle\right\rangle_{q}\right)+4 d_{e} d_{\mathrm{s}} g \Pi_{q}\left\langle\left\langle S^{z} \mid S^{z}\right\rangle\right\rangle_{q}+d_{\mathrm{s}}^{2}\left\langle\left\langle S^{z} \mid S^{z}\right\rangle\right\rangle_{q} .
$$

The expression for the susceptibility $\chi_{\mathrm{T}}$, obtained within the framework of the diagrammatic approach has a similar form, but the function $\left\langle\left\langle S^{z} \mid S^{z}\right\rangle\right\rangle_{q}$ is defined by the formula (24) instead of (36). We see that the static isothermal susceptibility, which is measured experimentally when a system is in equilibrium with the environment, does not coincide with the static isolated susceptibility.

\section{Instabilities and phase transitions}

Investigations of thermodynamically equilibrium and stable states of the PEM performed in the weak coupling case revealed (in the $\mu=$ const regime) the possibility of the phase transitions of the first or the second order both between uniform phases (with a jump of the pseudospin mean value $\eta$ and electron concentration $n$ ) and into the doubly modulated phase [21]. An analysis of the possibility of the other type of orderings (including the incommensurate ones) can be performed when one studies the behaviour of the static dielectric susceptibility $\chi_{\mathrm{T}}$ and obtains an information about the stability loss by investigating the singularity points of the function $\chi_{\mathrm{T}}$. The thermodynamic parameter values, at which $\chi_{\mathrm{T}} \rightarrow 0$, determine the spinodal points. The equation

$$
\lambda^{2}+g^{2} \sin ^{2} \theta \lambda\left\langle\sigma^{z}\right\rangle_{0} \Pi_{q}+\lambda^{2} g^{2} \beta b^{\prime} \cos ^{2} \theta \Pi_{q}=0
$$


was solved together with the equations for the mean values $\eta$ and $n$

$$
\begin{aligned}
& n=\frac{1}{N} \sum_{k \sigma}\left(\mathrm{e}^{\beta\left(g \eta+t_{k}-\mu\right)}+1\right)^{-1} \equiv \frac{1}{N} \sum_{k \sigma} f\left(E_{k}-\mu\right), \\
& \eta=\frac{h-g n}{2 \lambda} \tanh \left(\frac{\beta \lambda}{2}\right), \quad \beta=\frac{1}{T} .
\end{aligned}
$$

written in the MFA. The numerical calculations were used to calculate $\Pi_{q}$ (square lattice is considered, direct momentum summation is used; in our calculations we put $W=1)$.

At the fixed values of the chemical potential, the critical point can be defined as an upper point of spinodal (on the $(T, h)$ plane) with the highest temperature depending on the wave vector $\vec{q}$ value. Figure 1 shows the dependences of the critical temperature and the corresponding wave vector on the chemical potential in the case $\Omega=0$ (only positive values of the chemical potential are shown; at $h=g$, the picture is symmetrical with respect to the point $\mu=0$ which coincides with the centre of the energy band). We can see that the case $\vec{q}=(\pi, \pi)$ is realized when $|\mu| \lesssim 0.25$ at the chosen values of the parameters, which means that the system can pass into the phase with a doubly modulated lattice period. The case $\vec{q}=0$ (the transition into the uniform phase) is realized when $0.85 \lesssim|\mu| \leqslant 1.25(1.25=W+g / 2$; this value corresponds to the upper edge of the band when $\left.\left\langle S^{z}\right\rangle=1 / 2\right)$. The system undergoes the transition to the incommensurate phase at intermediate values of the chemical potential.

Figure 2 shows the dependences of the critical temperature and the corresponding wave vector on the chemical potential in the case $\Omega=0.2$. We can see that the presence of the tunnelling splitting narrows the interval of values of $\mu$ at which the above mentioned transitions take place; at high enough values of $\Omega$, the transition into the chess-board phase occurs only [21]. At the decrease of the pseudospinelectron coupling constant $g$, the interval of the values of $\mu$ at which the cases $q=\pi$ and $q=0$ are realized will become narrower.
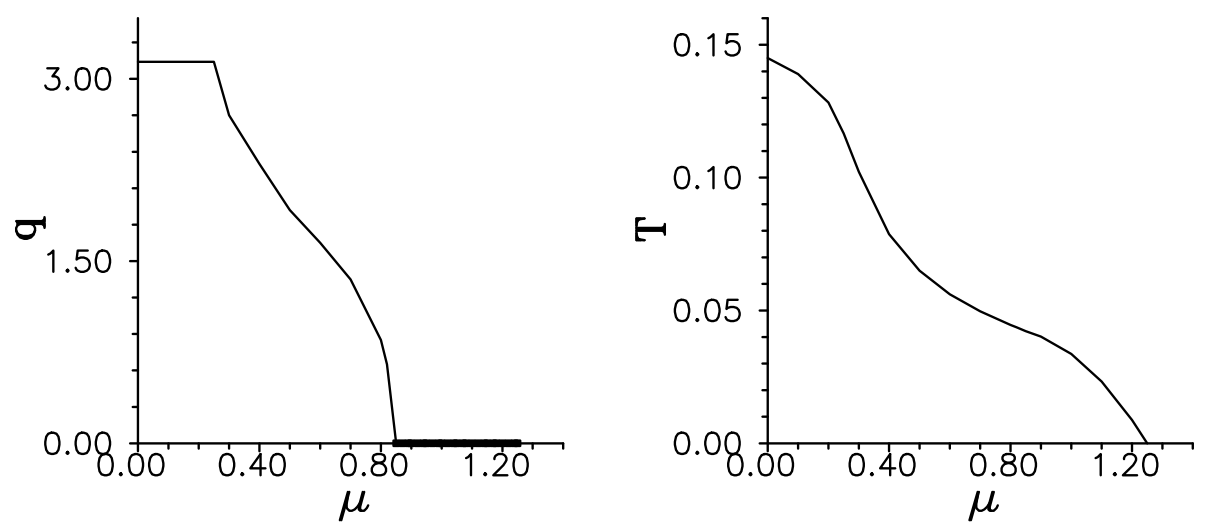

Figure 1. The dependences of the modulation wave vector $\vec{q}=(q, q)$ and the temperature of absolute instability of high-temperature phase on the chemical potential, $\Omega=0, g=0.5$. 

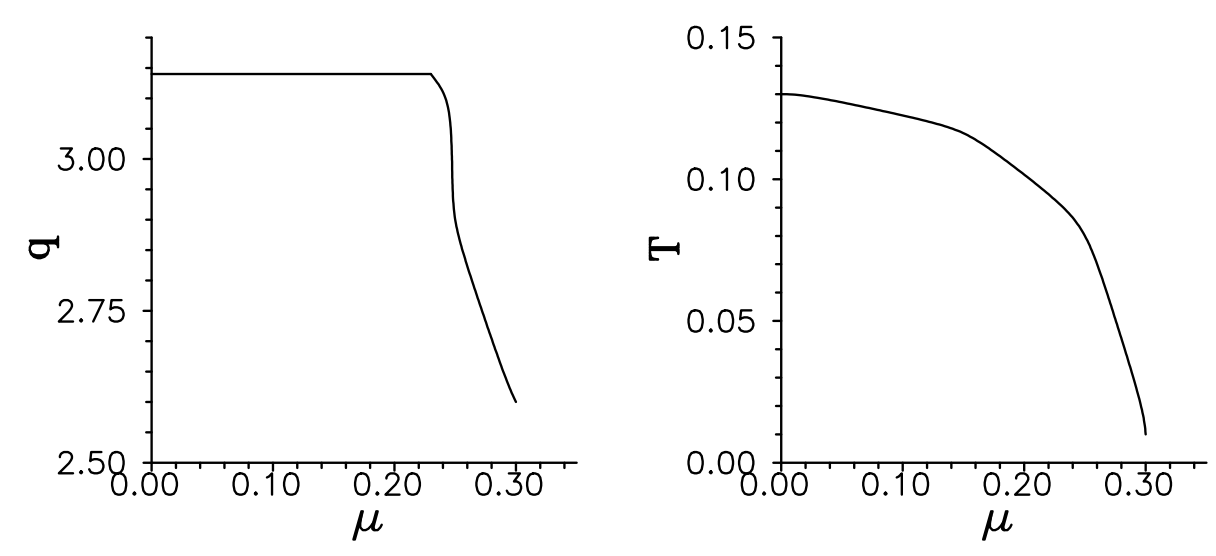

Figure 2. The dependences of the modulation wave vector $\vec{q}=(q, q)$ and the temperature of absolute instability of high-temperature phase on the chemical potential, $\Omega=0.2, g=0.5$.

The case $q=0$ was considered more in detail in the paper [21]. Using the set of equations (38) and the expression for the grand canonical potential

$$
\frac{\Phi}{N}=-\frac{T}{N} \sum_{k, \sigma} \ln \left(1+\mathrm{e}^{\left(\mu-t_{k}-g n\right) / T}\right)-T \ln \left(2 \cosh \frac{\beta \lambda}{2}\right)-g n \eta,
$$

it was shown that the system undergoes the first order phase transition between two uniform phases with jumps of the electron concentration and pseudospin mean value [21]. The phase transition line in the $(T, h)$-plane terminates in the above considered critical point. The line has a bent with respect to the $T$-axis which makes the phase transition possible with a change of temperature. The presence of the tunnelling-like splitting leads to the decrease of the phase transition temperature.

Let us consider now the case of double modulation of the lattice (the $q=\pi$ instability), when the crystal is divided into two sublattices. We introduce the notations: $\eta_{\alpha}=\left\langle S_{i \alpha}^{z}\right\rangle, n_{\alpha}=\sum_{\sigma}\left\langle n_{i \alpha \sigma}\right\rangle$ ( $\alpha=1,2$ is the sublattice index), $i$ is the unit cell index. The following set of equations for the electron concentrations and pseudospin mean values in sublattices is obtained in this case:

$$
\begin{aligned}
n_{1,2} & =\frac{1}{N / 2} \sum_{k \sigma}\left(\frac{1+\cos 2 \phi}{2}\left(\mathrm{e}^{\beta \lambda_{k(1,2)}-\mu}+1\right)^{-1}+\frac{1-\cos 2 \phi}{2}\left(\mathrm{e}^{\beta \lambda_{k(2,1)}-\mu}+1\right)^{-1}\right), \\
\eta_{\alpha} & =\frac{h-g n_{\alpha}}{2 \tilde{\lambda}_{\alpha}} \tan \left(\frac{\beta \tilde{\lambda}_{\alpha}}{2}\right) ; \quad \alpha \neq \beta .
\end{aligned}
$$

Here

$$
\begin{aligned}
& \sin 2 \phi=\frac{t_{k}}{\sqrt{\left(g \frac{\eta_{1}-\eta_{2}}{2}\right)^{2}+t_{k}^{2}}}, \quad \cos 2 \phi=\frac{-g \frac{\eta_{1}-\eta_{2}}{2}}{\sqrt{\left(g \frac{\eta_{1}-\eta_{2}}{2}\right)^{2}+t_{k}^{2}}}, \\
& \left.t_{k}^{11}=t_{k}^{22}=0, \quad t_{k}=t_{k}^{12}=t_{k}^{21}=\sum_{i-j} t \mathrm{e}^{\mathrm{i} \vec{k}\left(\vec{R}_{i 1}-\vec{R} j 2\right.}\right), \quad t=W / 4,
\end{aligned}
$$




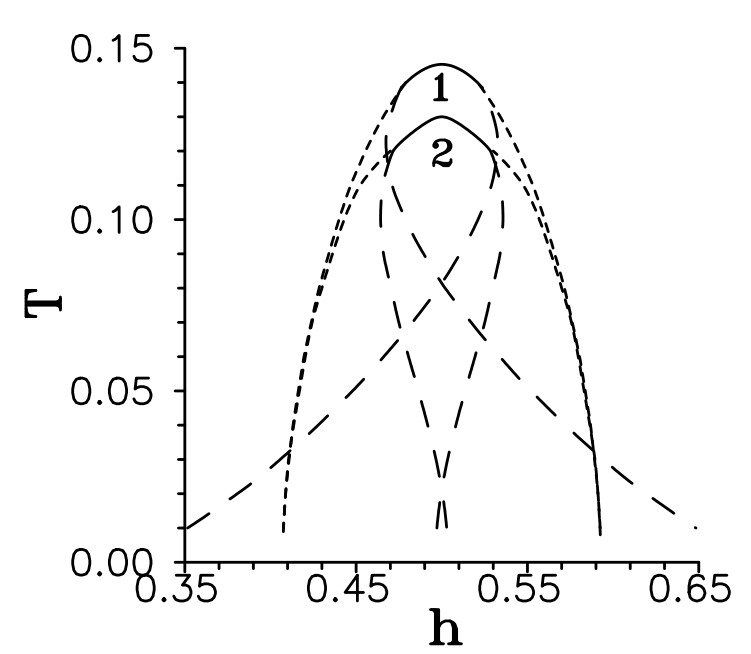

Figure 3. Spinodals (dashed lines) and the lines of the phase transitions (solid line denotes the second order phase transition, dotted line denotes the first order phase transition) from the uniform to doubly modulated phase $(1-$ $\Omega=0,2-\Omega=0.2 ; \mu=0, g=0.5)$.

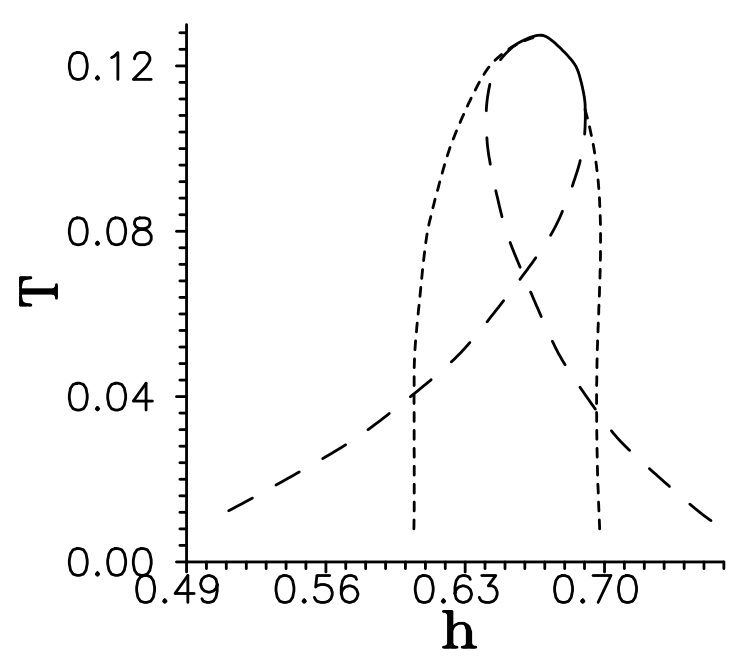

Figure 4. Spinodal (dashed line) and the line of the phase transition (solid line denotes the second order phase transition, dotted line denotes the first one) from the uniform to doubly modulated phase $(\Omega=0, \mu=0.2, g=0.5)$.

$$
\begin{aligned}
& \lambda_{k 1,2}=g \frac{\eta_{1}+\eta_{2}}{2} \mp \sqrt{\left(g \frac{\eta_{1}-\eta_{2}}{2}\right)^{2}+t_{k}^{2}}, \\
& \tilde{\lambda}_{1,2}=\sqrt{\left(g n_{1,2}-h\right)^{2}+\Omega^{2}} .
\end{aligned}
$$

The grand canonical potential in this case is given by the expression:

$$
\begin{aligned}
\frac{\Phi}{N / 2}= & -\frac{T}{N} \sum_{k} \ln \left(1+\mathrm{e}^{-\left(\lambda_{k 1}-\mu\right) / T}\right)\left(1+\mathrm{e}^{-\left(\lambda_{k 2}-\mu\right) / T}\right) \\
& -T \ln \left(4 \cosh \frac{\beta \tilde{\lambda_{1}}}{2} \cosh \frac{\beta \tilde{\lambda_{2}}}{2}\right)-g\left(n_{1} \eta_{1}+n_{2} \eta_{2}\right) .
\end{aligned}
$$

The phase transitions between uniform and doubly modulated (chess-board) phases, at which the difference $\eta_{1}-\eta_{2}$ (or $n_{1}-n_{2}$ ) plays a role of the order parameter, can be described by comparison of potential (39) and (42) and separation of the thermodynamically most stable states. The phase transition lines from a uniform to a doubly modulated phase are shown in figure 3 . The spinodal points are presented in this figure as well. This transition is of the second order (solid line) when the spinodal (dashed line) coincides with the transition line, otherwise the transition is of the first order(dotted line) (see [21]).

The increase of the tunnelling splitting leads to the decrease of the critical temperature. It can be shown (see [21]) that in the case of a square lattice, when the electron DOS possesses a logarithmic singularity in the centre of the energy band, 

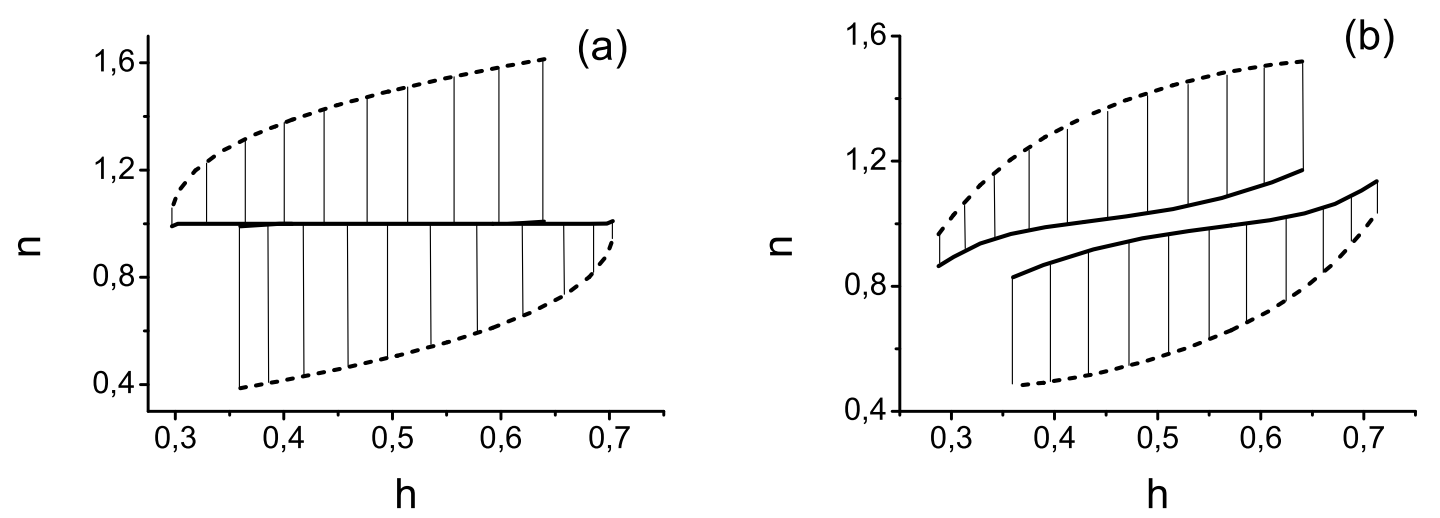

Figure 5. $(n, h)$ phase diagram, $\Omega=0$. Phase separation regions are shown for different temperatures: (a) $T=0.008$, (b) $T=0.08$. Dashed lines denote the borders of the uniform phase, thick solid lines denote the borders of the phase with doubly modulated lattice period.

there does not exist a critical value of $\Omega$ at which critical temperature is equal to zero; at large values of $\Omega$, the following expression for the critical temperature is obtained:

$$
T_{\mathrm{c}}^{*} \approx 2 W \exp \left(-\frac{\pi \sqrt{\Omega W}}{g \sqrt{2}}\right) .
$$

Figure 4 shows the phase transition line and spinodal in the case $\mu=0.2, \Omega=0$. Comparing with the case $\mu=0$, we can see that the picture is a asymmetric. There are two temperatures at which the phase transition changes its order (from the first to the second one). Lower temperature corresponds to the higher value of the field $h$.

The fact of the existence of the first order phase transitions between uniform and doubly modulated phases points to the possibility of separation into these two phases. It takes place at certain values of the electron concentration. The corresponding $(n, h)$ diagrams are shown in figure 5 . The chemical potential within the interval corresponds to the existence of the chess-board phase (see figure 1). The borders of the separation regions were obtained from the convexivity condition of the free energy defined as $F / N=\Phi / N+n \mu$. At the increase of temperature, the separation area narrows and at the high enough temperatures, the phase transition is of the second order and the phase separation region disappears.

\section{Spectrum of excitations}

The collective excitation spectrum of the system is described by the imaginary part of susceptibility $\chi_{\mathrm{I}}\left(\right.$ or $\left.\chi_{\mathrm{T}}\right)$ as a function of frequency and wave vector. We will consider the case when the electron energy band is almost empty: $\mu-g \eta \gtrsim W$, 
$T \rightarrow 0$. Let us find the more explicit expression for

$$
\Pi_{q}(\omega)=\sum_{k} \frac{n_{k}-n_{k-q}}{\omega+t_{k}-t_{k-q}}
$$

in this case. At the small values of $q$, we can expand in powers of $q$ the denominator of the expression for $\Pi_{q}$. One can obtain the following result for the real part of $\Pi_{q}$ in the cases $\omega>2 t k_{\mathrm{F}} q+q^{2} t$ and $\omega<-2 t k_{\mathrm{F}} q+q^{2} t$ (the imaginary part of $\Pi_{q}$ in these cases is equal to zero):

$$
\begin{aligned}
& \operatorname{Re} \Pi_{q}=\frac{k_{\mathrm{F}}^{2} q^{2} t}{2 \pi \omega^{2}}\left(\omega>2 t k_{\mathrm{F}} q+q^{2} t\right) \\
& \operatorname{Re} \Pi_{q}=\frac{k_{\mathrm{F}}^{2}}{2 \pi q^{2} t}\left(\omega<-2 t k_{\mathrm{F}} q+q^{2} t\right),
\end{aligned}
$$

here $k_{\mathrm{F}}=\sqrt{(W-g \eta+\mu) / t}-$ Fermi momentum. The imaginary part of the Green's function $\langle\langle M \mid M\rangle\rangle$ has a delta-peak when the denominator in (24) is equal to zero and $\operatorname{Im} \Pi_{q}=0$. The following expression is obtained for the frequency of this peak (which describes the pseudospin-wave excitations):

$$
\begin{array}{ll}
\omega_{p s}=\lambda-g^{2} \sin ^{2} \theta\left\langle\sigma^{z}\right\rangle \frac{k_{\mathrm{F}}^{2} q^{2} t}{2 \pi \lambda^{2}} & \left(\omega>2 t k_{\mathrm{F}} q+q^{2} t\right), \\
\omega_{p s}=\lambda-g^{2} \sin ^{2} \theta\left\langle\sigma^{z}\right\rangle \frac{k_{\mathrm{F}}^{2}}{2 \pi q^{2} t} & \left(\omega<-2 t k_{\mathrm{F}} q+q^{2} t\right) .
\end{array}
$$

In the frequency region $-2 t k_{\mathrm{F}} q+q^{2} t<\omega<2 t k_{\mathrm{F}} q+q^{2} t$ the imaginary part of $\Pi_{q}$ is different from zero and the delta-peak disappears. There exists a continuous electron excitation spectrum instead. Dispersion curves of the pseudospin-wave excitations and continuous excitation spectrum are shown in figure 6. Such a picture

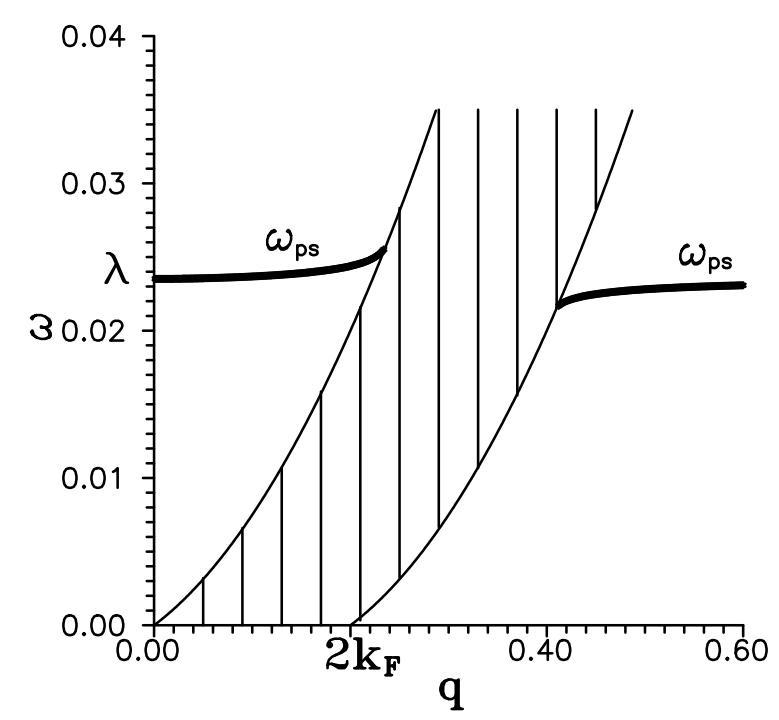

Figure 6. Excitation spectrum of the system. $\mu=1.22, \Omega=0.01, T=0.006$, $h \approx 1.02, k_{\mathrm{F}}=0.1$. 
of the spectrum is similar to that obtained for magnetic systems when the RPA is applied to investigate the ferromagnet with itinerant electrons described by the Hubbard Hamiltonian (see [25]). From the behaviour of magnetic susceptibility, the presence of spin-wave excitations and wide continuous spectrum of the Stoner-type excitations was deduced; these two spectra can superimpose. The similar picture is characteristic of the $s-d$ model (see [26]), here the itinerant electrons and localized electrons (spins) are taken into account. It was shown in the framework of the RPA that there exist two branches of spin-wave excitations (optical and acoustic ones) and the renormalized spectrum of Stoner-type excitations. The mechanism, which generally leads in the magnetic systems to the appearance of spin-wave or magnon excitations (sharp peak in the excitation spectrum) is connected with the interaction between the electrons and the interaction of electrons with spins. In the PEM, the mechanism is analogous: the appearance of the collective spin-like excitations is connected with the presence of pseudospins and their interaction is connected with conducting electrons.

\section{Conclusions}

The pseudospin-electron model in the case of weak pseudospin-electron coupling is considered in this paper in the framework of the approximation, which is of the type of the RPA (or GRPA). The analytical expressions for isothermal and isolated dielectric susceptibility are obtained. The points of absolute instability of hightemperature phase are found. It is revealed that similarly to the case of FK model the transition to another uniform phase occurs when the chemical potential is situated near the band edges. The transition to the phase with a doubly modulated lattice period takes place when the chemical potential is near the band centre. The incommensurate phase is realized at intermediate values of the chemical potential. The increase of the tunnelling parameter $\Omega$ narrows the interval of $\mu$-values at which the phase transitions take place. For the $n=$ const regime, the conditions of the appearance of the phase separation connected with the phase transitions between the uniform phase and the phase with doubly modulated lattice period are established.

It is interesting to compare the herein obtained results with the ones for the FK model at small values of the constant of the interaction between localized and itinerant particles. As it was mentioned in the introduction, in the case of the FK model, the possibility of the appearance in the system of the chess-board and segregated phases was revealed $[16,17]$; in [17] the presence of incommensurate phase was established. The incommensurate phase is realized at the intermediate values of the chemical potential of electrons and the chess-board and segregated phases are realized when the chemical potential is near the band centre and near the band edges, respectively. A similar picture is obtained here for the PEM. But, in contradiction, we consider in this paper the two dimensional lattice. This gives an advantage at the interpretation of the dielectric susceptibility divergences due to the explicit dependence of the $\chi_{\mathrm{T}}(\vec{q}, 0)$ function on the wave vector. In the $d \rightarrow \infty$ limit, such a dependence enters only through the function $X(q)=\frac{1}{d} \sum_{j}^{d} \cos q_{j}$ [16]; it leads to 
some difficulties at the consideration of incommensurate ordering. Besides, in $[16,17]$ the regime of the fixed concentration of localized particles was used; in the PEM this corresponds to the regime $\left\langle S^{z}\right\rangle=$ const. It is not inconceivable that the usage of such a regime led the authors to the conclusion that the transition to the chess-board phase at $g \lesssim W$ is always continuous (of the second order one) and the spinodals were considered to be the lines of the phase transition to the chess-board phase. We did not use here such a regime and analysing the behaviour of the grand canonical potential we showed that the transition to the chess-board phase can be both of the second order (the spinodals are the phase transition lines) and of the first order (spinodals do not coincide with phase transition lines). Due to the presence of the first order phase transition to the chess-board phase, the possibility of the phase separation on the uniform and chess-board phases in the case of weak coupling was demonstrated. Such a possibility, as was shown in [18], exists in the case of large values of a coupling constant. It should be mentioned that in [20] the possibility of phase separation on the different uniform phases was investigated only. Such a possibility was established for the PEM in our previous paper [21] at the consideration of the first order phase transitions between uniform phases. In contradiction to the FK model we take into account in the PEM the tunnelling-like splitting and investigate its role in the phase transition picture. We showed that its presence leads to the decrease of the phase transition temperature and to the narrowing of the region of the $\mu$ values at which phase transitions take place.

The phase transitions in the PEM at a weak coupling are similar in general to that revealed in this model in the case of a strong interaction, $g \gg W[13]$. However, the transition to incommensurate phase is not present at a strong coupling. This transition reveals itself only at the weak coupling. The physical mechanisms of transitions in these two cases are different; in the strong coupling regime the band is always split and the transition into the modulated phase or between the uniform ones is connected with the reconstruction of the electron spectrum in subbands while at a weak coupling the band remains unsplitted in the case of transitions between different uniform phases; the splitting of the initial energy band occurs only for the transition into the chess-board phase.

Analysing the behaviour of the dynamic susceptibility it was shown that there exists a smooth electron excitation spectrum besides the pseudospin-wave excitations. These two types of spectrum can superimpose one another. Such an excitation spectrum is similar to that revealed for magnetic systems, the appearance of sharp peak in the excitation spectrum is connected with the presence of pseudospins and their interaction with electrons.

\section{References}

1. Müller K.A. // Z. Phys. B, 1990, vol. 80, p. 193.

2. Hirsch J.E.,Tang S. // 1989, Phys. Rev. B, vol. 40, p. 2179.

3. Frick M., van der Linden W., Morgenstern L., Raedt H. // Z. Phys. B - Cond. Matt., 1990, vol. 81, p. 327. 
4. Stasyuk I.V., Shvaika A.M. // Acta Phys. Polon. A, 1993, vol. 84, p. 293.

5. Stasyuk I.V., Shvaika A.M., Schachinger E. // Physica C, 1993, vol. 213, p. 7.

6. Stasyuk I.V., Shvaika A.M. // Physica C, 1994, vol. 235-240, p. 2173; // Condens. Matter Phys., 1994, No. 3, p. 134.

7. Stasyuk I.V., Shvaika A.M., Danyliv O.D. // Mol. Phys. Rep., 1995, vol. 9, p. 61.

8. Stasyuk I.V., Velychko O.V. // Ukrainian Journal of Physics, 1999, vol. 44, p. 772

9. Stasyuk I.V., Havrylyuk Yu. // Condens. Matter Phys., 1999, vol. 2, p. 487.

10. Stasyuk I.V., Shvaika A.M. // Czech. J. Phys., 1996, vol. 46, p. 961.

11. Stasyuk I.V., Danyliv O.D. // Phys. stat. sol. (b), 2000, vol. 219, p. 299.

12. Stasyuk I.V., Shvaika A.M. // J. Phys. Stud., 1999, vol. 3, p. 177.

13. Stasyuk I.V., Shvaika A.M., Tabunshchyk K.V. // Condens. Matter Phys., 1999, vol. 2, p. 109; Ukrainian Journal of Physics, 2000, vol. 45, p. 520.

14. Izyumov Yu.A., Letfulov B.M. // J. Phys.: Cond. Matt., 1990, vol. 2, p. 8905.

15. Falicov L.M., Kimball J.C. // Phys. Rev. Lett.,1969, vol. 22, p. 997.

16. Brandt U., Mielsch C. // Z. Phys. B, 1989, vol. 75, p. 365; 1989, vol. 79, p. 295; 1989, vol. 82 , p. 37.

17. Freericks J.K. // Phys. Rev. B, 1993, vol. 47, p. 9263; 1993, vol. 48, p. 14797.

18. Letfulov B.M. // Eur. Phys. J. B, 1998, vol. 4, p. 447; 1999, vol. 11, p. 423.

19. Freericks J.K., Gruber Ch., Macris N. // Phys. Rev. B, 1999, vol. 60, p. 1617.

20. Freericks J.K., Lemanski R. // Phys. Rev. B, 2000, vol. 61, p. 13438.

21. Stasyuk I.V., Mysakovych T.S. // J. Phys. Studies, 2001, vol. 5, p. 268.

22. Gebhard F. The Mott Metal-Insulator Transitions: Models and Methods. Berlin, Springler-Verlag, 1997.

23. Izyumov Yu.A., Kassan-ogly F.A., Scryabin Yu.N. Field Methods in the Theory of Ferromagnetism. Moskow, Nauka, 1974 (in Russian).

24. Stasyuk I.V., Levytskii R.R. // Ukrainian Journal of Physics, 1974, vol. 19, No. 8, p. 1331 (in Russian).

25. White R.M. Quantum Theory of Magnetism. Moscow, Mir, 1972 (in Russian).

26. Kuz'min E.V., Petrakovskij G.A., Zavadskij E.A. Physics of Magnetically Ordered Matter. Novosibirsk, Nauka, 1976 (in Russian). 


\title{
Псевдоспін-електронна модель зі слабкою взаємодією
}

\author{
І.В.Стасюк, Т.С.Мисакович \\ Інститут фізики конденсованих систем НАН України, \\ 79011 Львів, вул. Свєнціцького, 1 \\ Отримано 17 травня 2002 p.
}

Досліджується статична та динамічна діелектрична сприйнятливість псевдоспін-електронної моделі в наближенні хаотичних фаз. В режимі $\mu=$ const виявлено можливість фазового переходу до однорідної фази, фази з подвійною модуляцією періоду ґратки та неспівмірної фази в залежності від значення хімічного потенціалу. Виявлено можливість фазового розшарування на однорідну фазу та фазу з подвоєнням ґраткового періоду та побудовано фазову діаграму $(n, h)$. Досліджено вплив тунельного розщеплення на фазові переходи. Встановлено присутність у системі псевдоспін-хвильових збуджень, неперервного спектру збуджень та можливость їх взаємоперекриття.

Ключові слова: псевдоспін-електронна модель, фазові переходи, спектр збудження

PACS: $63.20 . R y, 64.70 . K b, 71.10 . F d, 77.80 . B h$ 
